\title{
Endorsement of gender stereotypes in gender diverse and cisgender adolescents and their parents
}

\author{
Benjamin deMayo ${ }^{1}{ }^{*}$, Shira Kahn-Samuelson ${ }^{1}$, Kristina R. Olson ${ }^{1}$ \\ 1 Department of Psychology, Peretsman Scully Hall, Princeton, New Jersey, 08540 \\ * Corresponding author: bdemayo@princeton.edu
}

\begin{abstract}
Previous work has documented adolescents' gender stereotype endorsement, or the extent to which one believes men or women should embody certain traits. However, understanding of gender stereotype endorsement in gender diverse adolescents - those who identify with a gender different from their assigned sex at birth - is limited. Gender diverse adolescents' unique lived experiences with gender raise the question of whether they endorse gender stereotypes with the same frequency as cisgender adolescents. In this study, we investigated two primary research questions: (1) if gender diverse $(\mathrm{N}=$ $150)$ and cisgender $(\mathrm{N}=174)$ adolescents $(13-17$ years $)$ or their parents differed from one another in their endorsement of gender stereotypes; (2) the relationship between adolescents' and their parents' endorsement of gender stereotypes, and adolescents' predictions of their parents' endorsement of stereotypes. We found (1) no significant differences between gender diverse and cisgender adolescents in endorsement of gender stereotypes, perceptions of their parents' gender stereotype endorsement, and parents' gender stereotype endorsement, though parents endorsed stereotypes less than adolescents; and (2) adolescents' endorsement of gender stereotypes showed a weak positive association with their parents' gender stereotypes and the adolescents' predictions of their parents' stereotype endorsement, though neither correlation was significant. These results suggest that, in our sample, explicit stereotype endorsement was rare among gender diverse and cisgender adolescents and their parents.
\end{abstract}

\section{Introduction}

Gender is one of the most salient social categories, starting early in childhood and continuing into adulthood [1]. As a result, a large body of psychological research has set out to understand people's acknowledgement and endorsement of gender stereotypes, which are societally pervasive and highly consequential. In particular, psychologists have examined how adolescents, generally defined as young people between puberty and adulthood, conceptualize and endorse gender stereotypes [2], since adolescence is a pivotal time in social, emotional and sexual maturation [e.g., 3,4].

Almost all research on adolescents' gender stereotyping has studied cisgender people, or those whose gender identity (the gender they feel they are) matches the sex assigned to them at birth. Less is known about gender stereotypes in gender diverse adolescents (those whose gender identity does not align with their assigned sex, including binary transgender, nonbinary and gender nonconforming individuals), despite the growing number of youth identifying with this group. Is the development of gender stereotypes different in this population of young people different than in the cisgender samples that 
have typically been studied? The question is of both theoretical and practical import. Understanding how gender diverse adolescents conceptualize gender stereotypes could further our understanding of how one's own experience with gender (non)conformity relates to stereotyping. Further, given the increasing visibility of gender diverse youth in the public sphere, it is critical that their experiences be represented in research documenting the trajectory of gender development across the lifespan.

In the present study, we set out to answer this question by surveying a large sample of gender diverse adolescents on their endorsement of gender stereotypes using a previously validated measure [5]. Additionally, we collected data from a large sample of cisgender adolescents as a comparison sample in order to assess whether gender diverse adolesents' gender stereotyping differs from that of cisgender adolescents. Finally, to better understand the relation between parents' beliefs and children's beliefs, we assessed their parents' gender stereotyping, and surveyed adolescent participants on their expectations about their parents' or primary parents' stereotyping.

\section{Gender stereotyping in adolescence}

Prior research on gender stereotyping in adolescence has yielded mixed evidence on the extent of adolescents' gender stereotyping. Some researchers have found adolescence to be a time of life when gender roles intensify markedly [e.g. 6], and find, as a

consequence, that adolescents tend to rigidly endorse gender stereotypes, even more so than children in late elementary or early middle school [7]. Making a similar prediction, others posit that repeated reinforcement learning from the social environment regarding gender roles results in continued gender stereotype endorsement well into adolescence $[8,9]$. Conversely, some studies report an opposite trend of gender flexibility in adolescence $[10,11]$, while others find that substantial individual differences obscure any clear group-level pattern of gender stereotyping in adolescence $[12,13]$.

\section{Gender stereotyping in gender diverse youth}

Regardless of how one construes prior research on adolescents' endorsement of gender stereotypes, the findings cannot confidently be applied to gender diverse individuals. In fact, until relatively recently, little empirical work had examined how transgender youth of any age conceptualized gender stereotypes or whether they would endorse them in a meaningfully different way from cisgender peers. Three recent studies have, however, assessed gender stereotyping in transgender youth, their siblings, and matched cisgender participants.

These studies - all focused on younger children - show mixed evidence, but generally suggest cisgender and gender diverse children do not differ in their level of gender stereotyping. Three- to five-year-old transgender and cisgender children (siblings of transgender children and unrelated cisgender children) did not significantly differ in how much they thought men and women should engage in certain gender-stereotyped activities, as demonstrated in a paradigm adapted from Liben et al. (2002) [14]. Similarly, 6-11 year old transgender children, their siblings, and unrelated cisgender children did not differ significantly in their endorsement of prescriptive gender stereotypes; moreover, all groups of children tended to tolerate gender nonconformity [15]. However, a study focusing on gender stereotyping in 6-8 year old children found that transgender children and their siblings showed significantly lower levels of gender stereotype endorsement, and more willingness to socially affiliate with gender nonconformers, than the matched cisgender group [16]. In sum, preschool and elementary aged transgender children appear to endorse gender stereotypes at similar levels as their cisgender peers; when differences do appear the transgender group appears to show lower levels of stereotype endorsement and greater tolerance of gender 
nonconformity. Our investigation of adolescents' gender stereotyping thus adds another data point that can help elaborate any possible between-group differences in stereotype endorsement (or lack thereof).

\section{Parent influence on adolescents' endorsement of gender stereotyping}

In the current work, we are also interested in understanding whether adolescents' endorsement of gender stereotypes is associated with their parents' endorsement of gender stereotypes. Previous evidence has shown that parents' gender-related cognitions are associated with their children's. Notably, a meta-analysis [17] examined 43 studies investigating the link between parents' and children's gender schemas, and found that parents' gender-related attitudes about others were modestly associated with their children's. While the measures used, and psychological constructs assessed, in previous work vary considerably (see [18] for a review on measurement differences), past work generally indicates that parents may influence their children's thinking about gender; we were thus interested in seeing whether we would obtain a similar result when examining gender diverse adolescents' stereotype endorsements.

\section{Current work}

In our current work, we were interested in exploring the extent to which gender diverse adolescents endorse gender stereotypes, and how this may or may not differ from gender cisgender adolescents' gender stereotype endorsement. To do so, we recruited both a large sample of gender diverse adolescents as well as a group of cisgender adolescents, both of which completed a measure asking them to indicate how much they believed certain occupations, activities and traits should be practiced or held by men versus women. In addition, building on past work examining parental influences on children's gender stereotyping, we also asked their parents to complete the same measure, and asked adolescents to predict their parents' responses on the measure, in order to determine whether parents' stereotype endorsement (or adolescents' assumptions about parents' stereotype endorsement) was predictive of adolescents' responses on the same measure.

\section{Methods}

\section{Participants}

Participant families were recruited from different sources depending on whether they were intended to be included in the gender diverse group or the cisgender group.

\section{Gender diverse adolescents and parents.}

Most of the gender diverse adolescents in this study and their parents are participating in one of two larger longitudinal projects on gender development in U.S. American and Canadian transgender or other gender diverse children. The current measures were given as part of one wave of data collection. In order to expand the sample of gender diverse adolescents for this sample, some gender diverse youth were also recruited through email advertisements to listservs of professional organizations related to transgender health and well-being.

Participants were excluded if the variables of interest were not completed enough to be scored ( $\mathrm{N}=23$ parents, $\mathrm{N}=22$ adolescents), or if the participants spent too little time on the survey (less than 5 min, $\mathrm{N}=1$ adolescent; the median time to completion 
was approximately 24 minutes on the entire survey, which included other measures not directly related to the topic discussed in this paper). Participants who responded to the items of interest at all were included, even if they chose the 'skip' option on every item (as opposed to leaving the items entirely blank, in which case they were excluded). If participants accidentally submitted the survey more than once, their first

administration was used and the others were excluded $(\mathrm{N}=5$ parents, $\mathrm{N}=2$

adolescents). Finally, after data collection began, we noticed issues with data quality

coming from a small subset of participant recruited from online channels (rather than the participants drawn from the longitudinal study); we therefore reviewed all of these non-longitudinal participants and made determinations about who would be included vs. not without examination of the dependent variables. Instead, we based these exclusions off of inconsistencies or peculiarities in the participant responses that seemed unlikely to result from participant errors or difference in perspectives between adolescents and parents. For example, in deciding to exclude participants, we examined whether the youth's birthday was consistent across teen and parent consent forms, whether the developmental trajectory of pronoun use reported by parents and adolescents was roughly similar, and whether participants' reported trajectory of pronoun use was roughly compatible with the current gender identity and assigned sex reported on the form. As a result of these exclusions, 10 parents and 9 adolescents were excluded, leading to a sample of 178 parents and 150 adolescents in the gender diverse recruitment group. We did confirm, however, that all results from regression models discussed later in this paper hold if these latter participants are included.

\section{Cisgender adolescents and their parents.}

The comparison sample of cisgender adolescents and their parents were recruited from a database of participants maintained by the University of Washington Psychology Department. We collected 211 individual parent submissions and 187 individual adolescent submissions in the cisgender recruitment group. Of these, 12 parents and 8 adolescents were excluded for being blank or having missing data, and 4 parent submissions were excluded for being duplicate submissions from participants who had already responded, leaving us with 195 parent submissions and 177 adolescent submissions from the cisgender recruitment group.

\section{Final participant N's.}

In the vast majority of cases, adolescents from the gender diverse recruitment group were gender diverse, and adolescents from the cisgender recruitment group were cisgender. However, 6 adolescents from the gender diverse recruitment group identified as cisgender at the time of testing, and 8 adolescents from the cisgender recruitment group identified as transgender, gender nonconforming, or nonbinary, thus qualifying for our purposes as gender diverse at the time of testing. In subsequent analyses, we use adolescents' own gender identification at the time of the study to determine whether they are analyzed as part of the gender diverse group or as part the cisgender group. In all, we had 150 adolescent-parent dyads in the gender diverse group and 174 adolescent-parent dyads in the cisgender group. Additional demographic information about participants is included in Tables 1 and 2.

\section{Procedure}

Parents were sent the study materials via email. After giving consent for themselves and their children to participate, they completed the parent portion of the study. Adolescents could either complete their portion immediately after the parent was 
Table 1. Parent demographics

\begin{tabular}{l|l|l}
\hline & $\begin{array}{l}\text { Parents: Cisgender } \\
\text { group }\end{array}$ & $\begin{array}{l}\text { Parents: Gender } \\
\text { diverse group }\end{array}$ \\
\hline Race & $5(3 \%)$ & $3(2 \%)$ \\
Asian & $0(0 \%)$ & $2(1 \%)$ \\
Black & $13(7 \%)$ & $9(6 \%)$ \\
Hispanic/Latino & $33(19 \%)$ & $11(7 \%)$ \\
Multiracial/Other & $123(71 \%)$ & $122(81 \%)$ \\
White, non-Hispanic & $0(0 \%)$ & $3(2 \%)$ \\
No race reported & $1(1 \%)$ & $6(4 \%)$ \\
Yearly income & $3(2 \%)$ & $23(15 \%)$ \\
$<\$ 25,000$ & $13(7 \%)$ & $16(11 \%)$ \\
$\$ 25,001-\$ 50,000$ & $49(28 \%)$ & $42(28 \%)$ \\
$\$ 50,000-\$ 75,000$ & $102(59 \%)$ & $60(40 \%)$ \\
$\$ 75,001-\$ 125,000$ & $6(3 \%)$ & $3(2 \%)$ \\
$>\$ 125,001$ & 2.6 & 1.86 \\
Income not reported & & \\
Mean politics rating $(1=$ most & & \\
liberal, $7=$ most conservative) & &
\end{tabular}

Table 2. Adolescent participants by race/ethnicity

\begin{tabular}{l|l|l}
\hline & $\begin{array}{l}\text { Adolescents: } \\
\text { Cisgender group }\end{array}$ & $\begin{array}{l}\text { Adolescents: } \\
\text { Gender diverse } \\
\text { group }\end{array}$ \\
\hline Race & $5(3 \%)$ & $5(3 \%)$ \\
Asian & $0(0 \%)$ & $4(3 \%)$ \\
Black & $10(6 \%)$ & $8(5 \%)$ \\
Hispanic/Latino & $35(20 \%)$ & $25(17 \%)$ \\
Multiracial/Other & $124(71 \%)$ & $108(72 \%)$ \\
White &
\end{tabular}


finished on the same device, or they could opt to receive a follow-up email with the study materials. In either case, the parent completed their portion first so that they could consent to their own and their child's participation. The study procedure was approved by IRB protocol \#00001527 at the University of Washington.

The measure of interest was a lightly adapted version of the Trait subscale from the Occupations, Activities and Traits Attitude Measure (OAT-AM) devised by [5]. The OAT-AM asks whether participants thought men, women, or both men and women should have various traits. Ten traits were designated as stereotypically masculine (e.g., being good at math; being aggressive), ten as stereotypically feminine (e.g., crying a lot, being good at English), and five were gender neutral (e.g., study hard). Participants were asked to rate these traits on a 1 to 5 scale, with 1 indicating that only men should have the trait, 5 indicating that only women should have the trait, and 3 indicating that both women and men should have the trait. They could also skip any individual trait. For analysis, masculine traits were reverse-coded, so that a score of 1 on an item signifies gender stereotype endorsement that is incongruent with societal expectations, while a score of 5 signifies maximal gender stereotype endorsement congruent with societal expectations. Skipped items were excluded from analyses.

The OAT-AM carries several advantages which motivated its use in the current work. First, it is a short form that takes little time to complete and can be embedded in a larger study, as was the case here. Second, the scale is high in face validity, in that it probes participants directly on their endorsement of gender stereotypes; it also has good test-retest reliability [5].

Adolescents completed the measure twice: once as a measure of their own opinions, and a second time indicating their perceptions of their parents' likely responses. We investigated two primary research questions: (1) if gender diverse and cisgender adolescents or their parents differed from one another in their levels of gender stereotyping, and (2) the relationship between adolescents' and their parents' endorsement of gender stereotypes, and adolescents' predictions of their parents' endorsement of stereotypes.

Participants completed this measure as part of a larger survey that investigated a range of different topics (e.g., mental health, medical transition, etc). The present measure was included as a stand-alone measure and therefore its relation to any other measures, beyond the demographics reported in this paper, has not been assessed.

\section{Results}

\section{Group differences in stereotype endorsement}

All groups of adolescents and parents overwhelmingly did not endorse gender stereotypes in their responses: as shown in Figure 1, all groups indicated that "both men and women" should have the stereotypically masculine and feminine traits more than $80 \%$ of the time.

Nonetheless, we were interested in whether gender diverse adolescents or their parents endorsed gender stereotypes at differing levels from cisgender adolescents and their parents. We scored participants' responses on a 1-5 scale, with 1 indicating societally incongruent stereotype endorsement (e.g., women should be dominant) and 5 indicating societally congruent stereotype endorsement (e.g., men should be dominant). In SM1, we show an alternative method of analyzing the results in which we treat participants' responses as a binary variable, indicating whether or not they had declined to endorse each stereotype (i.e., did they select the ' 3 ' [neutral response] or did they select any other answer); we include this analysis to adhere more closely to the recommended scoring procedure recommended by [5]. The results are similar across 


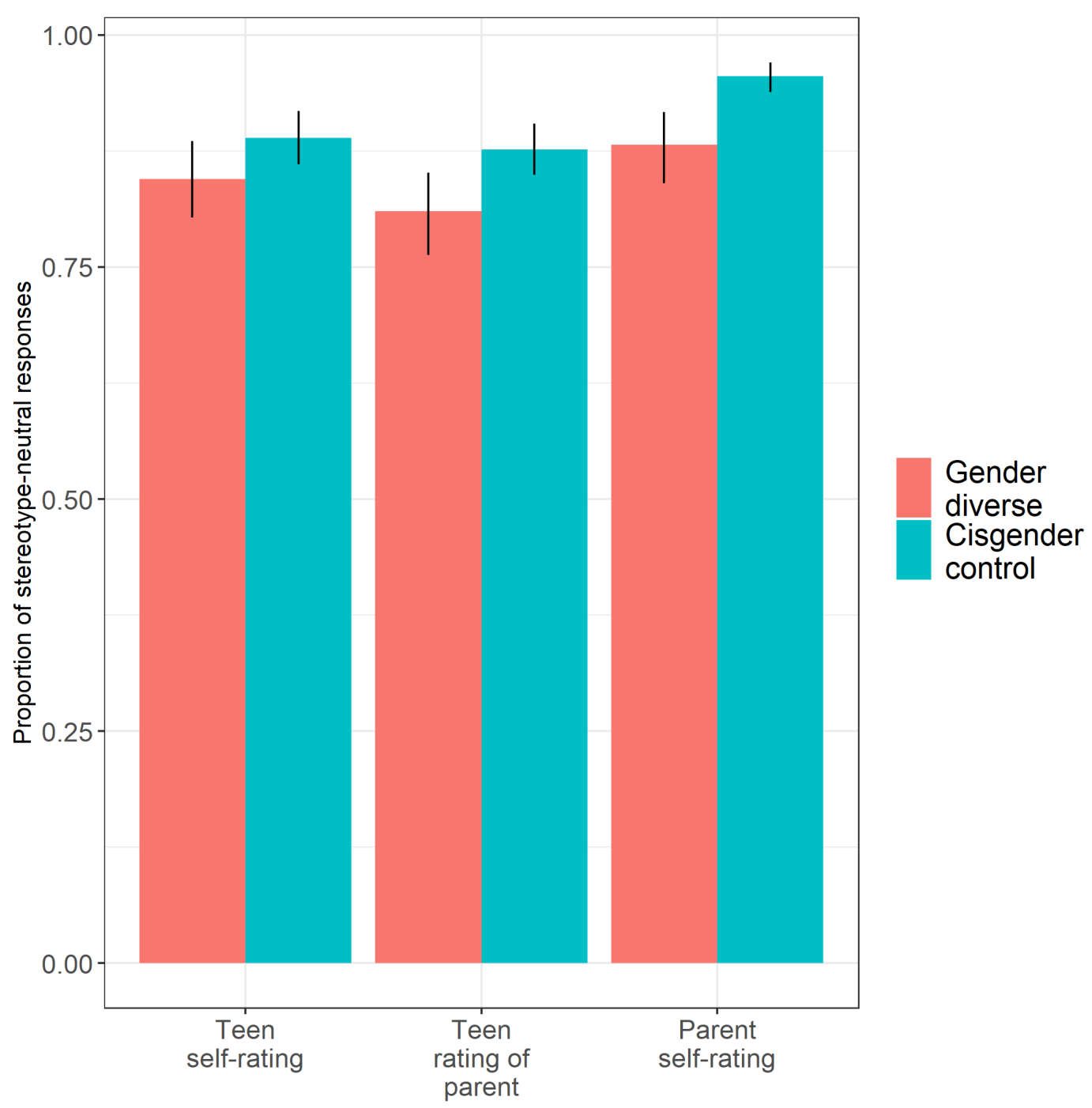

Fig 1. Proportion of non-stereotyped responses, averaging first within participants and then across participants, by gender identity condition (either Gender diverse, N = 150 dyads, or Cisgender, $\mathrm{N}=174$ dyads). 
Table 3. Regression output for main analysis

\begin{tabular}{l|r|r|r|r|c}
\hline Predictor & Estimate & Standard Error & df & t-value & p-value \\
\hline Intercept & 3.09 & 0.01 & 908.60 & 228.75 & $<.001$ \\
Question set: Adolescent guess & 0.01 & 0.02 & 640.11 & 0.84 & 0.402 \\
Question set: Parent self-report & -0.05 & 0.02 & 638.42 & -3.04 & 0.002 \\
Gender group: Gender diverse & -0.01 & 0.02 & 908.65 & -0.41 & 0.681 \\
Question set: Adolescent guess * Gender group: Gender diverse & 0.00 & 0.03 & 641.02 & 0.04 & 0.964 \\
Question set: Parent self-report * Gender group: Gender diverse & 0.02 & 0.03 & 638.54 & 0.73 & 0.463 \\
\hline
\end{tabular}

Table 4. Means, Standard Errors, and N's by Participant Group and Measure

\begin{tabular}{l|l|l|l|l}
\hline \multirow{2}{*}{ Measure } & \multicolumn{2}{|c|}{ Gender Diverse Group } & \multicolumn{2}{c}{ Cisgender Group } \\
\cline { 2 - 5 } & Mean (SE) & N & Mean (SE) & N \\
\hline Adolescent guess about the parent & $3.1(0.018)$ & 145 & $3.11(0.017)$ & 170 \\
Adolescent self-rating & $3.09(0.018)$ & 149 & $3.09(0.012)$ & 173 \\
Parent self-rating & $3.05(0.011)$ & 150 & $3.04(0.007)$ & 174 \\
\hline
\end{tabular}

either analytic approach.

We examined group differences in stereotype endorsement by fitting a linear mixed-effects model predicting participants' mean scores across items as a function of the gender identity of the adolescent in the dyad (gender diverse or cisgender), the particular question set (adolescent self-report, adolescent assumptions about the parent, and parent self-report), and the interaction between these factors. In order to account for the fact that each dyad has multiple mean scores (one for each question set), we included "random" intercepts for each unique dyad. Our final model specification was as follows: mean score question set * gender diverse identification of adolescent $+(1 \mid$ dyad), (where (1 | dyad) represents a random intercept term for each individual dyad.)

Tables 3 and 4 summarize the overall results. We found no significant differences in gender stereotype endorsement on the basis of gender identity; stereotype endorsement in participants from dyads in the gender diverse group did not differ from stereotype endorsement from those in the cisgender group, $\beta=-0.0082, p=0.681$. However, the mixed-effects regression model does show a significant main effect such that parents' responses are slightly lower in stereotype endorsement than adolescents', $\beta=-0.0532, p$ $=0.002$, corresponding to a reduction of 0.3 standard deviations in stereotype endorsement.

\section{Relationship between parents' and adolescents' endorsement of gender stereotypes}

Pair-wise partial correlations between mean scores on each question set were calculated, controlling for the score on the third question set. Parents' mean self-report scores showed a weak positive correlation with their children's mean self-report scores which was not statistically significant, Pearson's $r=0.106, t=1.888, p=0.06$. Mean scores of adolescents' assumptions about parents' stereotyping and the parents' actual stereotyping showed a weak positive correlation that was not statistically significant, Pearson's $r=0.083, t=1.478, p=0.14$. Of the three pair-wise partial correlations calculated, the strongest relationship emerged between adolescents' self-report and their assumption about their parents, Pearson's $r=0.239, t=4.347, p<.001$. 


\section{Discussion}

We used a previously-validated measure to assess gender stereotype endorsement in gender diverse and cisgender adolescents, as well as their parents. While endorsement of gender stereotyping in adolescence, and the impact of parents' gender cognition on their children's gender cognition, have been investigated previously $[17,18]$, to our knowledge no other work has studied gender stereotyping specifically in gender diverse adolescents and their parents.

Two main findings emerged. First, all groups of adolescents and parents showed very little endorsement of gender stereotypes (Figure 1). On nearly all items, nearly all participants endorsed the 'both' genders option (e.g., saying both men and women should be good at math). Parents endorsed stereotypes even less on average than adolescents. Among adolescents, we observed that gender diverse participants showed no significant difference in their endorsement of gender stereotypes as compared to cisgender participants. This finding converges with those in [14] and [15], in which gender diverse and cisgender children did not show differences in gender stereotyping, though differs from a prior study [16] which found lower levels of gender stereotyping in 6-8 year old transgender children. Second, adolescents' stereotype endorsement was showed a very weak positive association with their parents' stereotype endorsement. This result, which was not statistically significant, is reflective of the more general phenomenon described in [17] that parents' thinking about gender is, at most, modestly correlated with their children's.

Several limitations are present in the present work. First, the OAT-AM, while face valid and used widely the study of gender stereotyping, showed remarkably little response variability across participants; in fact, when indicating their own stereotype endorsement, over half of participants ( $61 \%$ of adolescents, $65 \%$ of parents) responded to every item on the scale (excluding skipped items) by saying that "both men and women" should embody the trait in question, meaning that all of the effects observed were driven by fewer than half the participants in the sample. The scale may have been too coarse to show more nuanced group-level differences in endorsement of gender stereotypes, or these may not have been the best example traits for assessing gender stereotyping at this particular moment in history. In future work, it may be more appropriate to use a measure that is less direct than the OAT-AM, since participants may be hesitant to explicitly deem certain traits as "man-like" or "woman-like." Additionally, our participant sample is also skewed towards white, upper middle- and upper-class people in the United States of America. As a result, the generalizability of these findings to a more representative sample of the U.S. population, or populations in other cultural or national contexts, is unknown.

\section{Conclusion}

We found that gender diverse adolescents and cisgender adolescents showed similar levels of endorsement of gender stereotype endorsement, suggesting that the experience of being gender diverse may not exert a strong influence on adolescents' propensity to endorse gender stereotypes. Adolescents' parents tended to show less gender stereotype endorsement than adolescents, but all groups' stereotype endorsement was low. To the extent that adolescents did endorse gender stereotypes, their stereotype endorsement showed a very slight but not statisically significant positive association with their parents' stereotype endorsement. These results contribute to a growing body of empirical work that aims to understand how an increasingly visible cohort of transgender, gender nonconforming and nonbinary youth engage with prevailing societal stereotypes about gender. 


\section{Acknowledgements}

We thank Robin Sifre and Dominic Gibson for their statistical guidance in preparing this manuscript. This work was supported by National Institute of Child Health and Human Development, HD092347 and National Science Foundation,

SMA-1837857/SMA-2041463 to K.R.O.

SM 1 Appendix. Stereotype endorsement as a binary variable.

SM 2 Table. Regression output: Stereotype endorsement as a binary variable.

SM 2 Appendix. Bayes Factor Analysis.

\section{References}

1.

Halim ML, Ruble D. Gender identity and stereotyping in early and middle childhood. Handbook of gender research in psychology. Springer; 2010. pp. 495-525.

2 .

Perry DG, Pauletti RE. Gender and adolescent development. Journal of Research on Adolescence. Wiley Online Library; 2011;21: 61-74. 3 .

Choudhury S, Blakemore S-J, Charman T. Social cognitive development during adolescence. Social cognitive and affective neuroscience. Oxford University Press; 2006;1: 165-174.

4.

Coleman JC, Hendry LB. The nature of adolescence. Psychology Press; 1999. 5 .

Liben LS, Bigler RS, Ruble DN, Martin CL, Powlishta KK. The Developmental Course of Gender Differentiation: Conceptualizing, Measuring, and Evaluating Constructs and Pathways. Monographs of the Society for Research in Child Development. 2002;67: i-183. Available: https://www.jstor.org/stable/ 3181530

Hill JP, Lynch ME. The Intensification of Gender-Related Role Expectations during Early Adolescence. In: Brooks-Gunn J, Petersen AC, editors. Girls at Puberty: Biological and Psychosocial Perspectives. Boston, MA: Springer US; 1983. pp. 201-228. doi $10.1007 / 978-1-4899-0354-9 \_10$

7.

Stoddart T, Turiel E. Children's Concepts of Cross-Gender Activities. Child Development. 1985;56: 1241-1252. doi:10.2307/1130239

8.

Power TG, Shanks JA. Parents as socializers: Maternal and paternal views. Journal of Youth and Adolescence. 1989;18: 203-220. doi:10.1007/BF02138801 9.

Richards MH, Gitelson IB, Petersen AC, Hurtig AL. Adolescent Personality in Girls and Boys: The Role of Mothers and Fathers. Psychology of Women Quarterly. 1991;15: 65-81. doi $10.1111 /$ j.1471-6402.1991.tb00478.x 
10 .

Katz PA, Ksansnak KR. Developmental aspects of gender role flexibility and traditionality in middle childhood and adolescence. Developmental Psychology. 1994;30: 272-282. doi:10.1037/0012-1649.30.2.272

11.

Carter DB, Patterson CJ. Sex roles as social conventions: The development of children's conceptions of sex-role stereotypes. Developmental Psychology. 1982;18: 812-824. doi: $10.1037 / 0012-1649.18 .6 .812$

12.

Crouter AC, Whiteman SD, McHale SM, Osgood DW. Development of Gender Attitude Traditionality Across Middle Childhood and Adolescence. Child Development. 2007;78: 911-926. doi https://doi.org/10.1111/j.1467-8624.2007. $01040 . x$

13.

Bogt TFM ter, Engels RCME, Bogers S, Kloosterman M. "Shake It Baby, Shake It": Media Preferences, Sexual Attitudes and Gender Stereotypes Among Adolescents. Sex Roles. 2010;63: 844-859. doi:10.1007/s11199-010-9815-1

14.

Fast AA, Olson KR. Gender Development in Transgender Preschool Children. Child Development. 2018;89: 620-637. doi:https://doi.org/10.1111/cdev. 12758

15.

Rubin JD, Gülgöz S, Alonso D, Olson KR. Transgender and Cisgender Children's Stereotypes and Beliefs About Others' Stereotypes. Social Psychological and Personality Science. 2020;11: 638-646. doi 10.1177/1948550619879911

16.

Olson KR, Enright EA. Do transgender children (gender) stereotype less than their peers and siblings? Developmental Science. 2018;21: e12606. doi https: //doi.org/10.1111/desc. 12606

17.

Tenenbaum HR, Leaper C. Are parents' gender schemas related to their children's gender-related cognitions? A meta-analysis. Developmental Psychology. 2002;38: 615-630. doi:10.1037/0012-1649.38.4.615

18.

Signorella ML, Bigler RS, Liben LS. Developmental Differences in Children's Gender Schemata about Others: A Meta-analytic Review. Developmental Review. 1993;13: 147-183. doi:10.1006/drev.1993.1007 


\section{Supplementary Material 1: Stereotype endorsement as a binary variable}

In addition to the analysis we presented in the main text in which we considered each response to be on a continuous 1-5 scale, we also analyzed participants' responses as a binary variable, indicating whether, on each item, they indicated that a particular trait should be held by both men and women (akin to choosing a response of " 3 " on the above continuous scale), or whether they chose a different response (any response other than "3"). Accordingly, we fit a separate mixed-effects generalized linear model with a logit linking function in which the dependent measure was the proportion of responses in which participants did not endorse stereotypes, and the predictors were the gender diversity of the child (cisgender comparison vs. gender diverse) and the question set (adolescent self-report, adolescent assumptions about the parent, and parent self-report), and the interaction between these two predictors. Additionally, we included a random intercept for each dyad. Thus, this analysis matches the main analysis presented in the Results section, with the adjustment that the outcome variable is a proportion between 0 and 1, as opposed to a mean score between 1 and 5 .

The full regression output is shown in Table SM1. The results described here are transformed into expected probabilities for easier interpretation. Overall, the predicted probability of the reference group (cisgender children self-reporting) choosing a non-stereotyped response were quite high (predicted probability $=0.957$ ).

As in the main analysis, there was a significant main effect of question set, such that parent self-report responses were associated with increasing the probability of choosing a non-stereotyped response from 0.957 in the adolescent self-report measure to 0.985 in the parent self-report measure, on average, conditional on all other model covariates.

Additionally, a significant interaction of gender diversity and question set emerged, such that in the gender diverse group, the difference in probability of choosing a non-stereotyped response when the parent was self-reporting (probability of non-stereotype endorsement $=0.967$ ) relative to when the child self-reporting (probability of non-stereotype endorsement $=0.948$ ) was mitigated. In other words, the gap between adolescents and parents was smaller in the gender diverse group than it was in the cisgender group.

In sum, parents in both groups were more likely to choose non-stereotyped responses to the survey items than their children, though the effect was very small and all groups were overwhelmingly likely to choose non-stereotyped responses.

Table SM 1: Regression output: Stereotype endorsement as a binary variable (all estimates are odds ratios)

\begin{tabular}{|c|c|c|c|c|}
\hline Predictor & Estimate & Standard Error & z-value & p-value \\
\hline Intercept & 3.09 & 0.18 & 16.73 & $<.001$ \\
\hline Question set: Adolescent guess & -0.13 & 0.08 & -1.57 & 0.12 \\
\hline Question set: Parent self-report & 1.12 & 0.11 & 10.41 & $<.001$ \\
\hline Gender group: Gender diverse & -0.19 & 0.26 & -0.71 & 0.48 \\
\hline Question set: Adolescent guess * Gender group: Gender diverse & -0.17 & 0.12 & -1.35 & 0.18 \\
\hline Question set: Parent self-report * Gender group: Gender diverse & -0.65 & 0.14 & -4.46 & $<.001$ \\
\hline
\end{tabular}




\section{Supplementary Material 2: Bayes Factor Analysis}

As an exploratory analysis, we also computed Bayes Factors in order to quantify, given the data observed, the evidence in favor of a null hypothesis (i.e., that the population difference in mean scores between the gender diverse and cisgender comparison groups is zero) over an alternative hypothesis (that the population difference in mean scores is not zero) for each of the three measures assessed (adolescent self-report, adolescent assumptions about the parent, and parent self-report). We first averaged scores within participants and measures, so that adolescents each had one mean score on the adolescent self-report measure and the adolescent assumptions about the parent measure, and parents each had one mean score on the parent self-report measure. We then used the BayesFactor package in R (Morey et al., 2015) to calculate the ratio between the marginal likelihoods of the null and alternative hypotheses, where the prior distribution of the standardized mean difference between groups varies according to a Cauchy distribution with scale parameter $\sqrt{1 / 2}$ under the alternative hypothesis, and the standardized mean difference between groups under the null hypothesis is assumed to be 0 . On the adolescent self-report scale, we obtained a Bayes Factor of 0.1322, indicating that the observed data were 7.563 times more likely under the null hypothesis than the alternative. On the adolescent assumptions about the parent measure, we obtained a Bayes Factor of 0.1296, indicating that the observed data were 7.717 times more likely under the null hypothesis than under the alternative. Finally, on the parent self-report measures, we obtained a Bayes Factor of 0.1751, indicating that the observed data were 5.71 times more likely under the null hypothesis than under the alternative.

\section{References}

Morey, R. D., Rouder, J. N., Jamil, T., Urbanek, S., Forner, K., \& Ly, A. (2015). Package 'bayesfactor'. http://cran/r-projectorg/web/packages/BayesFactor/BayesFactor.pdf. 